\title{
Crustal deformation and $A E$ monitoring: annual variation and stress-soliton propagation
}

\author{
M. Poscolieri ${ }^{1,2}$, G. P. Gregori ${ }^{1,2}$, G. Paparo ${ }^{1,2}$, and A. Zanini ${ }^{1,2,3}$ \\ ${ }^{1}$ Istituto di Acustica "O.M. Corbino", C.N.R., Via Fosso del Cavaliere 100, 0133 Rome, Italy \\ ${ }^{2}$ ICES - International Centre for Earth Sciences, Via Fosso del Cavaliere 100, 0133 Rome, Italy \\ ${ }^{3}$ Department of Mechanical Engineering, University of Rome "Tor Vergata", Via Politecnico 1, 00133 Rome, Italy
}

Received: 13 July 2006 - Revised: 23 October 2006 - Accepted: 23 October 2006 - Published: 3 November 2006

\begin{abstract}
The stress propagation through the crust can be effectively monitored by means of acoustic mission $(A E)$ techniques (ultrasounds). The $A E$ intensity is indicative of the amount of stress that affects some lithospheric and/or crustal slab of some (ultimately unknown) scale size. In principle, such scale size can be different in different areas, as it depends on their respective tectonic setting, by which a variety of prime causes ought to be considered: every cause can eventually prevail over others. Two basic phenomena are here reported. If the crust behaves like a comparatively ideal elastic body, an annual variation is observed, which appears in-phase and correlated, when comparing $A E$ records collected at the Italian site and on the Cephallonia Island. It seems being astronomically modulated, hence it should display a planetary scale. One likely explanation is in terms of the loading tide. Such interpretation can be confirmed by some additional modelling and analysis upon considering the motion of the Sun and of the Moon. A second observed effect refers to the case in which the crust feels the effect of the fatigue that reduces its elastic performance. The phenomenon can be described in terms of stress solitons that cross the area being monitored. They can be unambiguously recognised, and the possibility is therefore envisaged of eventually using them for measuring the propagation speed of stress through the crust over continental or planetary scales. The residuals, with respect to such regularly recognisable effects, of the recorded $A E$ signals are to be investigated in a few subsequent analyses (in progress), as they appear to contain additional relevant physical information, still being much different from any simple random noise. A final recommendation ought therefore to be stressed, for setting up some array of at least a few $A E$ recording stations to be simultaneously operated over some continental scale area and for a few years at least. Their potential applications still appear much promis-
\end{abstract}

Correspondence to: $\mathrm{M}$. Poscolieri

(maurizio.poscolieri@idac.rm.cnr.it) ing and to be still focused in their complete, specific, operative and physical details and interpretation.

\section{Introduction}

Acoustic emission $(A E)$ provides twofold information. On the one hand, the $A E$ intensity, averaged over some suitable time interval, is indicative of the amount of stress that affects some crustal slab of some (ultimately unknown) scale size. In principle, such size can be different in different areas, as it depends on their respective specific tectonic setting. A first concern deals therefore with the assessment of such typical scale size for a given area in order to manage, to match and to model, in some way, the available $A E$ observations and tectonic morphology.

The second kind of information provided by $A E$ refers to the fatigue of materials, which is characterised by the typical temporal sequence of the released $A E$ signals. Such aspect can be quantitatively investigated by fractal analysis of the $A E$ time series. It is likely to give relevant information for monitoring the temporal evolution of the system, in terms of its increasing fatigue, preceding, either on some long time range, or immediately, the final "catastrophe" of the system (e.g. a seismic shock).

A fundamental concern deals with the monitoring efficiency, as, in general, every $A E$ release is weak, and it soon damps off close to its emitting source, thus hardly reaching the $A E$ detector. In such a respect, however, two basic physical arguments ought to be taken into account.

A first constraint requires the existence of some adequately "solid" object. In fact, an "ideal solid", i.e. a "perfectly elastic" body, is an abstraction, which is only approximately matched by physical reality. Such "solid" body should act like an effective guide for channelling the $A E$ release from the $A E$ source through the $A E$ detector. Such configuration, in general, is very unlikely to occur in actual reality.

Published by Copernicus GmbH on behalf of the European Geosciences Union. 
A second physical argument deals with the space-scale of the prime causes that determine the microdeformations within every given "solid" object. For instance, suppose that we want to monitor the temperature variations of some given physical system, and suppose that we use some dense array of measured points. The temperature variation of the entire body is such that the records at different sites of the array must be physically correlated, as the body of concern is a unique physical system, and every monitored space domain is a part of one whole physical system that behaves according to some rationale in response to some common control factors. Hence, just by a matter of physics, the temperature records at different sites must result correlated with one another. Similarly, suppose that several glasses, more or less filled with wine, are on a table. Whenever the table is shaken, the wine within every glass shall start oscillating. If we detect the wine oscillation within one glass alone, we can well presume that the wine within every other glass shall oscillate. By this, nobody will claim that all glasses are connected with one another by some tubes, and that they compose a unique wine container, etc. Indeed, when we monitor one glass, no teleconnection occurs of the observed wine oscillation within any other glass. Rather, we state that every single glass is one independent detector of the trembling of the entire table. The glass certainly only reveals the trembling of the site where it is located. However, we can reasonably refer to one such detector for inferring the trembling of the entire table.

Concerning $A E$ monitoring, the $A E$ measured at one site is monitored by an acoustic transducer - which detects the $A E$ that are released reasonably close to the detector, and such physical distance depends on how "solid" is the body to which the transducer is applied. For instance, as a standard, the $A E$ transducer is applied over some rocky outcrop, which extends underground much like a dedicated probe, having some large, though unknown, spatial extension. Whenever some crustal slab is shaken around such probe, the stress distribution inside such huge solid body is changed, e.g. due to its own weigh. Whenever some crystal bonds yield, the "solid" body releases $A E$, which in general can be originated even at some comparatively close distance from the $A E$ detector; if the body is "solid" such distance can even be larger. Suppose that some large set of instruments is operated within some given region, and that it includes several such different outcrops. Every outcrop is to be conceived like an independent detector of the stress, which eventually crosses some given crustal slab - much like every wine glass is a detector independent of the other glasses or detectors. If some large crustal slab trembles, due to some crustal stress propagating through it, we shall detect several $A E$ transducers being activated in some correlated way. In principle, under some lucky circumstances, we can even expect that the time delay between the activation of the different $A E$ detectors can eventually give a measure of the propagation speed of the crustal stress (see below).
Summarising, there is no need for any teleconnection, and the $A E$ signal can even damp off within some imperfect "solid" body. In fact, we always detect, in any case, a local phenomenon. However, it is just a matter of an unquestionable and clear observational evidence that different sites being monitored eventually do reveal $A E$ records that result intrinsically physically correlated with one another. Therefore, we claim that we detect a crustal stress propagating through the crust. In the case of the Italian peninsula, we claim that we do recognise $A E$ signals preceding by several months an earthquake with epicentre located somewhere within some wide region, even at several hundred kilometres distance from the $A E$ recorder. By this, we do not claim that such large distances are connected by any waveguide for conveying $A E$ propagation. We rather assert that the entire crustal slab of the Italian peninsula is simultaneously shaken in some way, manifesting a correlation of the $A E$ release occurring over some large distance. That is, the frequently considered concept of local monitoring of some seismic or geodynamic activity - or claiming that we do detect phenomena strictly occurring very close to the location of the epicentre, and never at some large distance from it - must be abandoned, appearing just nonsense for a realistic interpretation of the physics of phenomena. In such a respect, when dealing with the time domain, several investigations in the literature report about the search for precursors that ought to anticipate the shock by some very long time advance. In contrast, apparently they never considered possible long-distance correlated crustal effects. Such choice certainly derived from the presumption of the correctness of some paradigm, while a much more realistic and understating approach had certainly to take into account the (ultimately unknown) observable spatial range of the stress phenomena being involved.

Our $A E$ records were operated typically within two frequency bands, respectively, around $180 \div 200 \mathrm{kHz}$ (called high frequency, or $H F A E$ ), and around $25 \mathrm{kHz}$ (low frequency, or $L F A E$ ). Concerning data acquisition and handling, refer to Paparo and Gregori (2003), Gregori and Paparo (2004), Paparo et al. (2006) and to Poscolieri et al. (2006). Every measurement is the integral of the rms $A E$ bursts, measured during a time span of $5 \mathrm{~ms}$. Every recorded signal is the average over $30 \mathrm{~s}$ of such $5 \mathrm{~ms}$ measurements. The signal is filtered by a specific procedure for rejecting outliers, which reflect phenomena not of concern for the present investigation (refer to Gregori and Paparo, 2004, for details). The resulting time series is smoothed by applying a weighted running average, over a $24 \mathrm{~h}$ time span, by using a triangular filter (with a maximum weight 1 at the centre, decreasing linearly to 0 ). This is the database for the subsequent analyses. The final result ought to exclude both, and get rid for the thermoelastic effects associated with the diurnal temperature cycle (see Paparo et al., 2002), and for some large fraction (though not all of them) of the diurnal tidal effects (see below). Such filtered datum ought therefore to be representative of the amount of stress that is crossing some large crustal 
area around the $A E$ recording site.

For clarity purposes, the rationale of the data analysis ought to distinguish among four items.

A previous paper (Gregori et al., 2005) was concerned with the first item, i.e. with an analysis dealing with the timedelayed correlation between $A E$ and seismic energy release. That is, the $A E$ records can be considered like earthquake precursors, although upon considering the aforementioned large regional viewpoint, opposed to any strictly local interpretation. The earthquakes that interested the area here considered are listed in Table 1, borrowed after Gregori et al. (2005).

The second item of concern is the object of the present study. It deals with the background $A E$ trend, independent of the precursors that are likely to be correlated with any earthquake. This is the analysis of interest for inferring observational evidence for the crustal stress propagation.

The third item is focused on the residual of the original database with respect to the aforementioned average general trend. The amplitude of such residual, however, results correlated with the intensity of the average trend, and such behaviour denotes an intriguing feature that seems likely to be associated with the specific mechanism of crustal stress generation and propagation (in preparation).

The fourth item refers to the newly evaluated remaining residual, when all previous recognised physical effects have been subtracted. It results not being a random noise. Hence, it is not a usual error-bar of any general given measurement. The central limit theorem cannot apply, and such new residual has no normal distribution. Rather, it contains some relevant physical information, related to the fatigue of the "solid" materials that are going to yield and release $A E$. Such fourth item is to be considered in a subsequent study (in preparation). Refer to Paparo et al. (2006).

\section{The long-range variation}

Figures 1, 2, 3, and 4, partly borrowed from Gregori et al. (2005) though including some relevant updating, show, respectively, the $H F A E$ and $L F A E$ 24-h average records for the Raponi site (at Orchi, Foligno, in Umbria, central Italy; see a map in Gregori et al., 2005) and for the Cephallonia Island (western Greece). Some remarkable and comparably more evident features, which resulted to be seemingly associated with seismic activity, are the concern of the analysis carried out by Gregori et al. (2005). An $A E$ crisis, i.e. a large and almost abrupt increase of the signal, was observed at the Raponi site, some $~ 7-8$ months in advance for $H F A E$, and some $\sim 2$ months in advance for $L F A E$, compared to the Molise earthquake, which had an epicentre at say $\sim 400 \mathrm{~km}$ distance. A similar feature, not here shown, had been observed within records collected close to Potenza in southern Italy and being a precursor of the Colfiorito earthquake (see Table 1). That is, the aforementioned rationale of a large re-

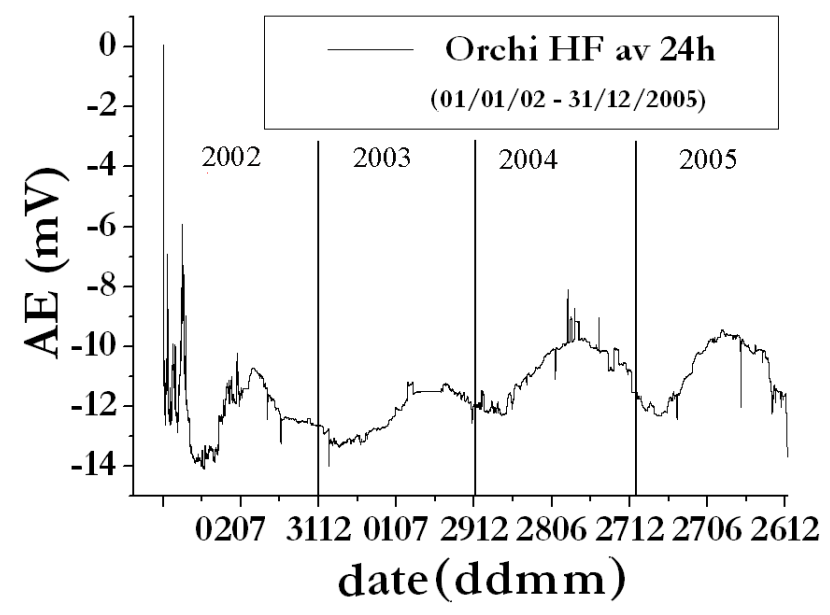

Fig. 1. Raponi site (Orchi, Foligno, central Italy). $H F A E$, weighted running average over $24 \mathrm{~h}$. The sharp feature on the left hand side is interpreted as a precursor of the Molise earthquake, occurred $~ 7-$ 8 months later, at about $400 \mathrm{~km}$ distance from the $A E$ recording site. Seismic phenomena do not appear to be local features. Rather they imply crustal stress crises that involve areas equal to, or maybe even larger than, the entire Italian peninsula. An annual modulation is also recognisable. See text. The acronym "ddmm" points out, respectively, day and month of that year.

gional shaking, in contrast with a mere local rationale, seems to be fairly well supported by such different and independent inferences, although both applying to the Italian peninsula, while it has to be expected that areas with a different tectonic setting behave differently.

In addition, such entire inference appears fairly well consistent with the expectation that $H F A E$ are very likely to be associated with the yield of comparatively smaller flaws within the crust, which later coalesce into flaws of increasingly larger size while they release $A E$ of progressively lower frequency - it is just the same elementary principle by which a violin cord of longer length vibrates at a comparatively lower frequency (Paparo and Gregori, 2003).

Some analogous evidence was investigated for the Cephallonia Island. The remarkably different tectonic setting, compared to the Italian peninsula, apparently plays a crucial role in determining some substantial different behaviour (e.g. see Poscolieri et al., 2006). In particular, such area is tectonically very complicated, and even the observed apparent times of the $A E$ precursors result to change in every case history (see Gregori et al., 2005). The physical system appears exceedingly complicated for being monitored by one $A E$ recording site alone. Some array of $A E$ recorders ought to be operated within some wider area (see also below).

The $A E$ trend of the background, independent of such anomalous occurrences, is the concern of the present study, i.e. independent of the eventual occurrence of the "catastrophe" of the system. In fact, a "catastrophe" occurs whenever the system experiences some irreversible large structural 
Table 1. Parameters of a few earthquakes.

\begin{tabular}{|c|c|c|c|c|c|c|}
\hline Name & date & $\begin{array}{l}\text { starting time } \\
\quad(\text { GMT })\end{array}$ & $\begin{array}{l}\text { latitude of epicentre } \\
\qquad(N)\end{array}$ & $\begin{array}{l}\text { longitude of epicentre } \\
\qquad(E)\end{array}$ & $M$ & $\begin{array}{l}\text { depth } \\
(\mathrm{km})\end{array}$ \\
\hline \multicolumn{7}{|c|}{1997 Colfiorito (1997; only shocks with $M \geq 5.0$ ) } \\
\hline \multirow[t]{6}{*}{1997 Colfiorito } & $26 \mathrm{Sep}$ & 003312.89 & $43^{\circ} 01.38$ & $12^{\circ} 52.42$ & 5.6 & 7.0 \\
\hline & $26 \mathrm{Sep}$ & 094026.73 & $43^{\circ} 01.78$ & $12^{\circ} 50.09$ & 5.9 & 8.0 \\
\hline & $03 \mathrm{Oct}$ & 085522.02 & $43^{\circ} 01.95$ & $12^{\circ} 49.90$ & 5.0 & 5.7 \\
\hline & 06 Oct & 232453.23 & $43^{\circ} 00.76$ & $12^{\circ} 49.79$ & 5.4 & 7.4 \\
\hline & $12 \mathrm{Oct}$ & 110836.87 & $42^{\circ} 54.13$ & $12^{\circ} 56.10$ & 5.1 & 2.6 \\
\hline & $14 O c t$ & 152310.61 & $42^{\circ} 54.11$ & $12^{\circ} 54.75$ & 5.5 & 5.5 \\
\hline \multicolumn{7}{|c|}{ Molise (2002) } \\
\hline Molise & $31 \mathrm{Oct}$ & 1132 & $41^{\circ} .76$ & $14^{\circ} .94$ & 5.4 & \\
\hline
\end{tabular}

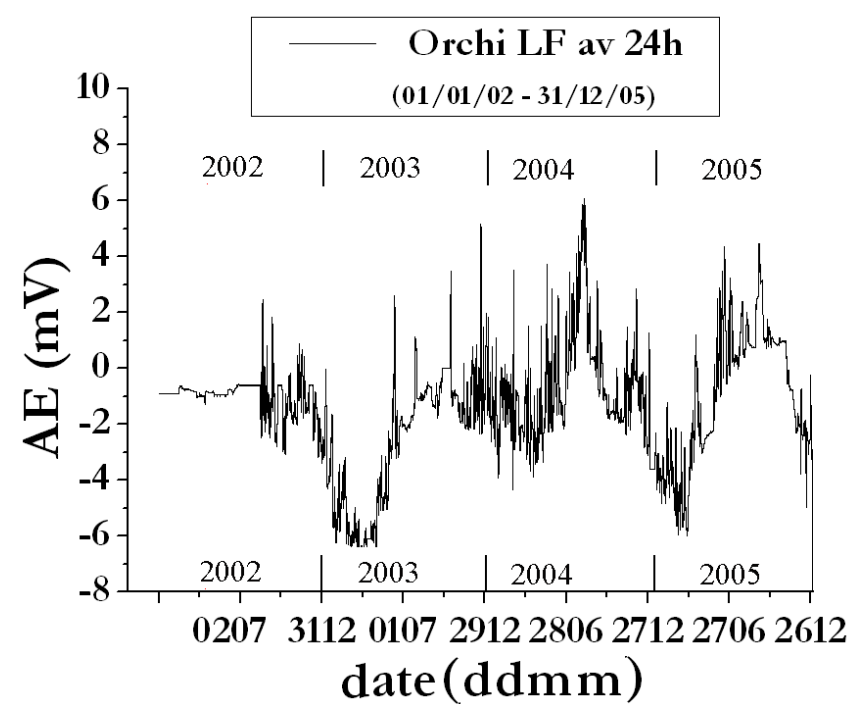

Fig. 2. The same as Fig. 1, but referring to $L F A E$. The abrupt change of trend, in terms of the introduction of some abrupt noise, preceded by almost exactly 2 months the Molise earthquake. The annual variation, if it exists, appears very confused and perturbed by some large noise. See text. The acronym "ddmm" points out, respectively, day and month of that year.

change, normally implying the release of some devastating amount of energy. In contrast, the smooth background denotes some regular process affecting the crustal slab, independent of the eventual yield of its rheological features.

The Raponi site was the epicentre of the Colfiorito earthquake. The $A E$ recording started at such site after the occurrence of such event. Figure 1 shows the large $H F A E$ crisis that preceded by $\sim 7-8$ months the Molise earthquake. Whenever such large anomaly is neglected, an annual variation is clearly recognised, and some sharp perturbations observed on the top of the second last maximum (from 1 to 30 of August 2004) presumably denotes some crustal stress per- turbation that crossed its entire surrounding area, almost like some short-lived soliton (see below).

Some equivalent perturbation is shown in Fig. 2 ( $L F A E)$, where, however, the annual variation definitely appears much more perturbed. Such inference denotes that the annual variation is associated with a phenomenon occurring during some comparatively much more preliminary stage of crustal stress propagation, when only the $H F A E$ are affected. But, when the physical system evolves, and some crystal bonds of comparatively larger size release $L F A E$, the system shall appear much more perturbed by the erratic local contribution of the fatigue of the crustal materials. Thus, the primary apparently regular annual modulation of the $H F A E$ shall result confused by some apparently erratic perturbation in the background. That is the signal-to-noise ratio for the large-scale crustal stress propagation turns out to get much worse when the $A E$ frequency is lowered. In any case, the most striking feature of Fig. 2 is the abrupt and sharp change of trend occurred on 15 August 2002, i.e. 77 days before the Molise earthquake. Such impressive feature is just a matter of fact, it is just one objective observation, and its physical interpretation is ultimately behind our present complete physical understanding. Only $A E$ records collected within some larger array of $A E$ recording sites, and a suitable consideration of the specific tectonic morphology of the entire Italian peninsula, could perhaps provide us with some objective physical input for attempting an actual significant interpretation.

Figures 3 and 4 are the analogous plots for Cephallonia. The $H F A E$ shows a clear annual variation, while, compared with Fig. 2, Fig. 4 appears much different. On the other hand, several evidences, and in particular the different behaviour on the occasion of different earthquakes occurred in that area (refer to Gregori et al., 2005, and to Poscolieri et al., 2006), clearly envisage the crucial role being played by the substantial difference of tectonic setting, when comparing the Italian peninsula with the Cephallonia environment. We have to consider seismic stress phenomena occurring within some 


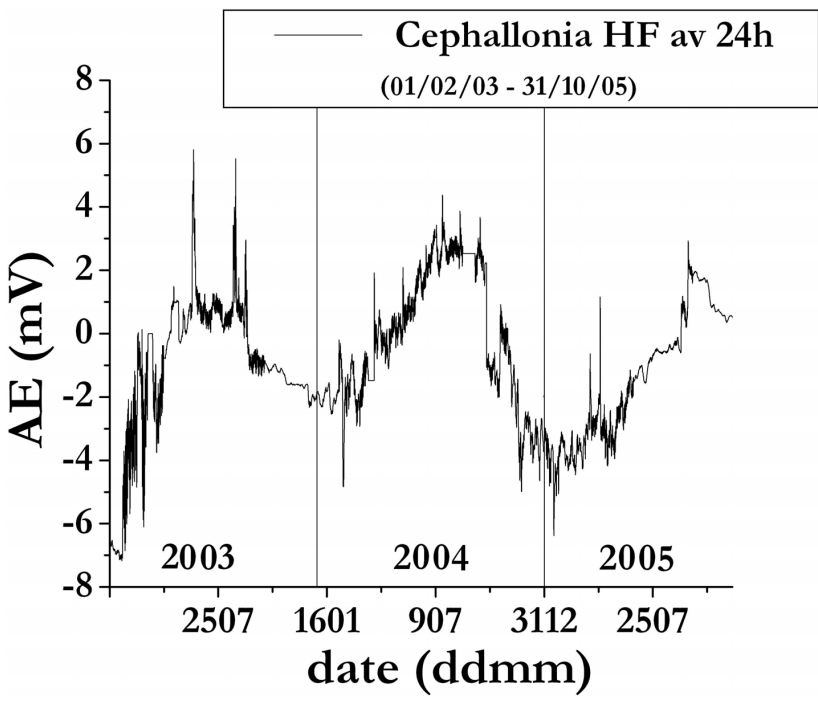

Fig. 3. Cephallonia Island. $H F A E$, figure analogous to Fig. 1. The annual variation appears very clear. The acronym "ddmm" points out, respectively, day and month of that year.

large region, and forget about the standard way of dealing only with local occurrences and observations. Different phenomena can be physically different case histories.

A striking feature of Fig. $4(L F A E)$ is the strong crustal perturbation crossing the Cephallonia area, just like one stress soliton, propagating through Cephallonia from the middle of April 2004 through the very beginning of 2005 . Upon a closer inspection of the entire available time span, perhaps some reminiscence of an annual trend can be recognised, although displaying some large interannual variation of the maximum amplitude of the oscillation. That is, the annual maximum appears more like a soliton lasting several months, better than a consequence of a repetitive and regular astronomical forcing. That is, the interannual change of the tectonic setting produces a substantially different amplification of the prime effect. Only a longer series of records can give justice of such tentative inference. $L F A E$ records appear certainly more perturbed than $H F A E$ records.

In any case, the $H F A E$ shows a clear annual variation, which is the concern of the next section. Its regularity envisages some astronomical control. In contrast, $L F A E$ appears to respond to some rationale that is substantially other than any mere astronomical control. Therefore, $H F$ and $L F A E$ ought to be interpreted according to comparatively different rationales, as $H F A E$ and $L F A E$ are intrinsically concerned with different phases in the evolution of the physical system. An eventual astronomical trigger can be eventually observed only when the system is not yet heavily biased by its internal fatigue, by which it responds like an effective and comparatively efficient elastic body. In contrast, when the system suffered by some degrading consequent to its fatigue, its elastic performance is reduced, and other effects result clearer, obscuring the former astronomical modulation.

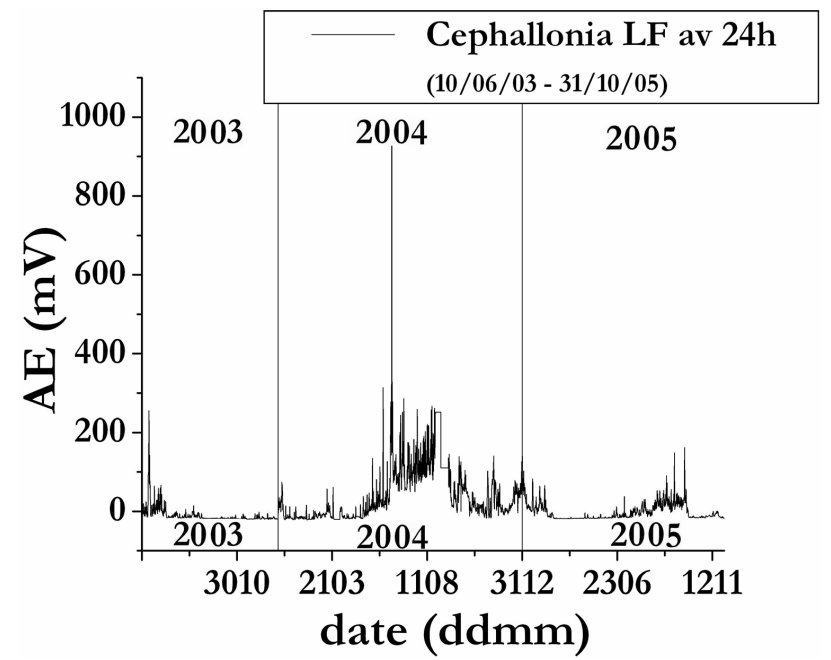

Fig. 4. Cephallonia Island. $L F A E$, figure analogous to Fig. 2. Compared to the case history of Fig. 2, the observed trend appears completely different. In general, a conspicuous crisis of crustal stress crosses the area. It appears just like one well defined soliton lasting from about April 2004 through the beginning of 2005. A new soliton (or some other perturbation) could have been more recently started. The entire area was interested by some conspicuous seismic activity during such soliton crossing. The tectonic setting is completely different from the Italian peninsula. No reliable interrelation can be guessed, due to the limited time series of available records, and due to the lack of any adequate array of simultaneously operated $A E$ recording sites. See text. The acronym "ddmm" points out, respectively, day and month of that year.

We have found some observational evidence and we do propose a physical interpretation. Its viability must, however, require additional observational tests, carried out by arrays of $A E$ recorders operated within some large area, and being eventually specifically implemented for such purpose.

We have therefore to deal with two effects. The astronomical modulation is the concern of the next section. Concerning the soliton evidence, it results to be clearly distinguishable from the background. Therefore, an eventual soliton ought to be clearly recognisable at different sites on the Earth, and its propagation speed could therefore be eventually measured whenever some array of $L F A E$ recording sites become available.

\section{The annual variation}

The annual variation, independent of its physical explanation, appears to be a clear effect. Owing to the reasons discussed in the preceding section, the signal-to-noise ratio appears much better for $H F A E$ compared to $L F A E$. Such basic physical drawback derives from the perturbation caused by other effects, which include tectonic occurrences, such as the stress perturbation crossing the region of concern, being eventually well defined and organised into solitons (see 


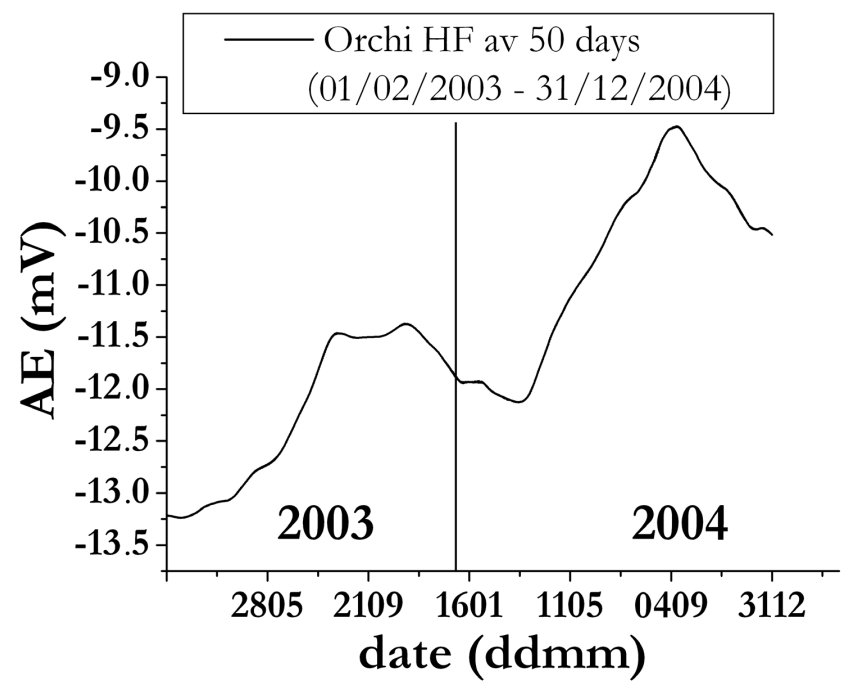

Fig. 5. Moving average, weighted by a triangular weigh, over 50 days, for the $H F A E$ records of the Raponi site records. The acronym "ddmm" points out, respectively, day and month of that year.

the previous section). That is, the increasing fatigue of the crustal materials originates $L F A E$ signals that are physically different from the $H F A E$ signals associated with the (yet unknown) causes of such regular annual variation. That is, the fatigue is not a drawback, as it permits detecting an effect, which is different from the astronomical modulation, and which can be even more interesting under several respects. Different phenomena sum up in giving the final recorded $A E$ signal. No reason requires that either one of such phenomena always prevails over the others. In general, under different circumstances, the cause of the annual variation prevails over the "perturbation" originated by other causes. In contrast, on other circumstances, such "perturbation", which was formerly considered "negligible", cancels any clear evidence for the annual variation. Upon suitable consideration of such physical expectations, the annual variation was therefore investigated as follows.

Owing to the reasons mentioned in the previous section, the concern deals with the $H F A E$, while the $L F A E$ have to be excluded, due to their excessive perturbation by the fatigue of the materials. The $24 \mathrm{~h}$ smoothed $H F A E$ signals of Figs. 1 and 3 have been smoothed by applying a weighted running average, by using a triangular weight of time-width 50 days.

Figure 5 shows the result for the Raponi site. The annual variation is very clear, although superposed over an increasing trend, which denotes that some crustal stress variation, of some longer period, crossed that area, and its physical cause was certainly other than the origin of the annual variation. Owing to the previously mentioned reasons, it appears therefore reasonable guessing that some long lasting soliton is crossing the Italian area. It could be stated that some large (continental) scale stress perturbation affected the Ital-

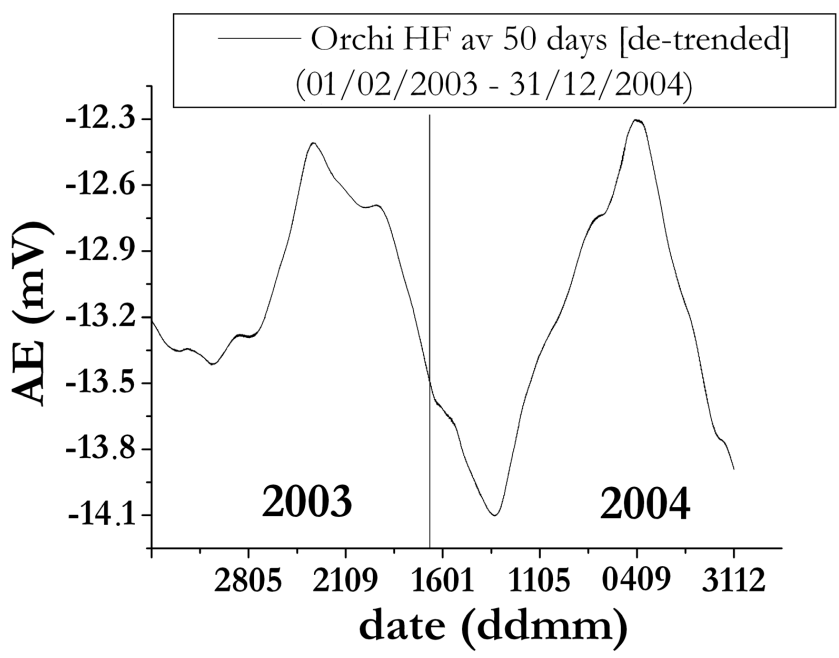

Fig. 6. The same as Fig. 5, after applying a linear de-trending. The acronym "ddmm" points out, respectively, day and month of that year.

ian peninsula. Confirmation of such guess ought to require consideration of a much longer $H F A E$ database. In addition, such possibility could be realistically checked and much better discriminated only whenever an array of $H F A E$ recording sites be eventually available. In any case, as far the present study is concerned, it is possible getting rid of such drawback. Figure 5 was therefore de-trended by subtracting a linear trend, thus giving Fig. 6. Figure 7 shows the analogous result for Cephallonia, where no de-trending appeared necessary.

The concern shifted therefore on the search for the eventual time-shift between the annual variations recorded at the Raponi site, and at Cephallonia. A convenient way of investigating one eventual such relative phase shift is based on a diagram, where on the abscissa and ordinate axis, respectively, the 50 day smoothed datum for the Raponi site and for Cephallonia are plotted. If the two signals are in phase, the plotted points must lay along a straight line. If they have some relative phase shift, they should look like one Lissajous figure - which is a well known classical concept from the history of physics, reported by several college textbooks on classical kinematics.

Figure 8 shows the result, which unquestionably shows that during 2004 the annual variation resulted clearly in phase at both sites. However, when putting together 2003 and 2004 the result appears much perturbed, as it is shown by Fig. 9 that includes both the perturbed 2003 and the regular 2004. The available database can give no evidence for any different phase shift or for any other apparently regular behaviour holding during 2003. It appears rather that during 2003 some different long-term effects biased any possible evidence of the regular annual variation. It appears much like the perturbation of some soliton that affected even the $H F A E$. An observational test for such guess could be given either by 


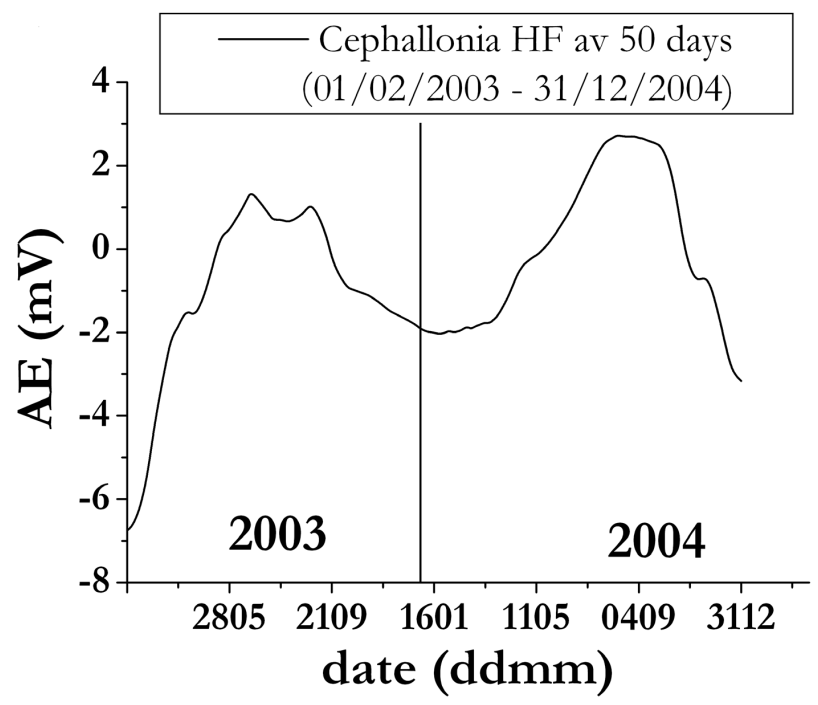

Fig. 7. The same as for Fig. 5, but dealing with the Cephallonia $H F$ $A E$ records. The acronym "ddmm" points out, respectively, day and month of that year.

operating an array of $A E$ recorders, or by using some higher frequencies $A E$ in order to reduce the bias of the fatigue of the materials that apparently affected even our $H F A E$ records during 2003.

\section{Discussing the physical interpretation of the annual variation}

Our available observations appear therefore consistent with an annual modulation of the crustal stress, which results simultaneously affecting both the Italian peninsula and the Cephallonia Island area. Notwithstanding the comparatively close location - compared to the planetary scale - of such two $A E$ recording sites, it appears that the conspicuous difference in their respective tectonic settings, and the greatly complicated tectonic pattern of Cephallonia, are in favour of a phenomenon that could reasonably be of planetary scale. Such large scale must be expected for every phenomenon that is controlled by some astronomical modulation. While waiting for the availability of the measurement carried out in some suitable set of additional $A E$ recording sites spanning continental size areas, we can attempt at giving some physical interpretation of our presently available observations.

For such a purpose, we have to appeal to some observational evidence being available by other techniques, and revealing some planetary scale control, or some astronomically correlated fact, that could originate some corresponding stress propagation through the crust. In fact, two such possible effects at least can be envisaged.

One physical fact is well known since a few decades. It derives from the astronomical determination of the increase of the length of the day (1.o.d.), and from the standard measure-

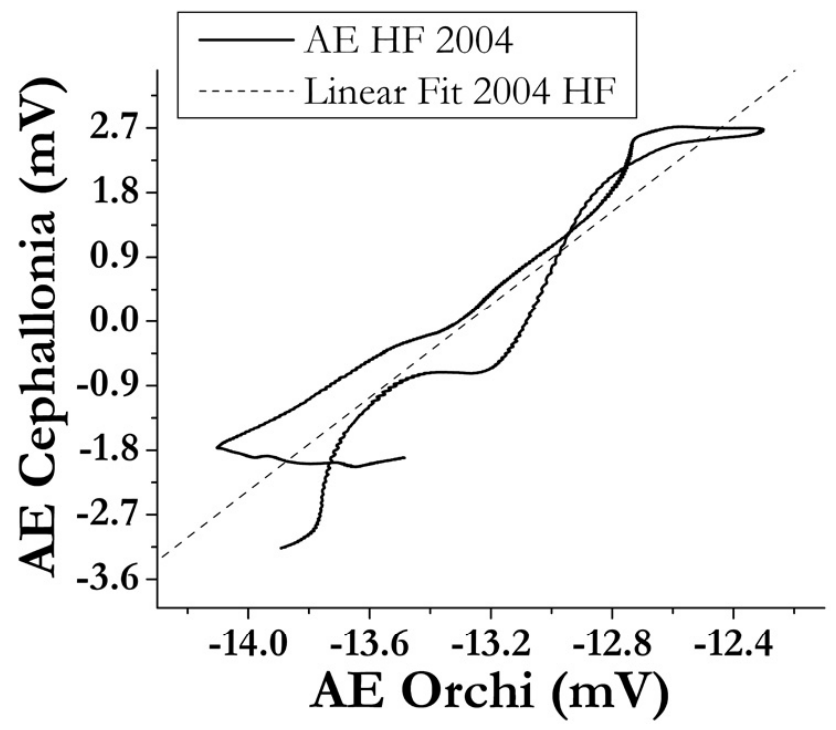

Fig. 8. Lissajous figure for 2004, plotting on abscissas the Raponi site 50 day-averaged $H F A E$ datum after de-trending, and on ordinates the Cephallonia 50 day-averaged $H F A E$ datum with no detrending.

ments of Earth's tides observed within some dense network of observatories covering the entire Earth. The regular increase of the 1.o.d. is interpreted as the effect resulting from the tidal pull, which is not, however, the direct consequence of the tide acting on the body of the planet Earth. Rather, it is now well acknowledged being the result of the so-called "loading tide". That is, the tide is much more effective on the ocean water than on the solid Earth, due to the much different viscosity, by which water can be easily displaced, unlike the solid components of crust, lithosphere and mantle. The displaced ocean waters finally press on the continental shelves, thus determining the torque that slows down the spin rate of the Earth. Such applied torque acts therefore only on the most external layers of the Earth's body. Hence, one must expect that some very intense stress must cross the Earth's crust. The power spent by tidal friction on the solid Earth is $\sim$ two orders of magnitude smaller than the tidal action on the ocean waters (see Gregori, 2002, Table B-1b for references). Wunsch (2000) quotes $\sim 0.310^{13} \mathrm{~W}$ for the energy loss in the Earth-Moon system, $\sim 0.210^{13} \mathrm{~W}$ of which is the total tidal dissipation energy, half of which occurs in open ocean by interaction with the ocean floor, and half controls the large-scale thermohaline circulation.

Let us consider the annual modulation of such effect, which, in principle at least, must be reasonably expected. We are not concerned about its existence. Rather, we should be concerned about the capability of measuring it by means of some apparatus, which should be proved having a sufficient sensitivity and precision for detecting it.

The tide is mainly of lunar origin, while only a smaller tide, about $1 / 5$ th of the lunar tide, is originated by the Sun. 


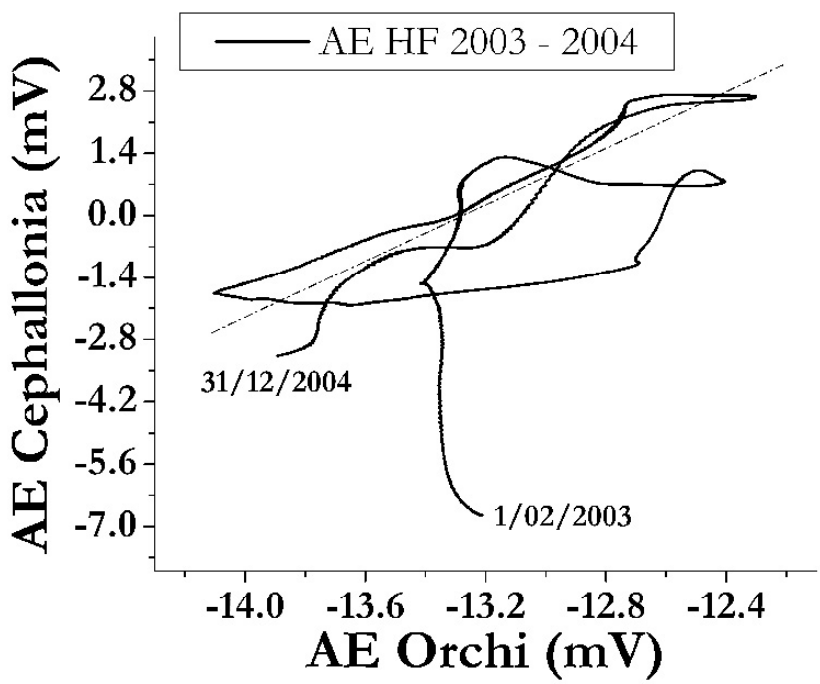

Fig. 9. The same Lissajous figure as for Fig. 8, but referring to both 2003 and 2004. The clear linear trend found for 2004 is lost in 2003, being the likely consequence of some long-trend soliton of crustal stress crossing the area. The fatigue of the crustal materials is the reason for the lack of a correct elastic response that should justify the ideal trend observed in 2004. See text.

For simplicity purposes, let us first take into account the solar tide.

We have to consider the ideal intersection of the Earth's body with the ecliptic plane (Fig. 10). Consider the largest ocean on the Earth, i.e. the Pacific Ocean. At every given date, let us "cut" its entire water extension, by planes parallel to the ecliptic plane and being regularly spaced compared to one another. That is, in this way let us divide the Pacific Ocean water into strips of some regular (e.g. constant) latitudinal extension. Let us apply the solar tide to every such strip of ocean water. Integrate the tidal pull that act on every such strip, and that pushes it on the continental shelf of Eurasia. Carry out the sum of such loading tide associated with all such strips. This is the resulting loading tide acting on the Eurasian continental shelf, derived from the tidal pull acting on the entire mass of the Pacific Ocean water.

Such effect can be computed for every date of the year. In general, it shall result different for different dates, depending on the different inclination of the Earth's spin axis with respect to the ecliptic plane. Such annual modulation can be computed. If the annual modulation of the crustal stress observed in the Mediterranean area is in phase with such computation, we can guess that such evidence is in favour of such speculated explanation. If not, we have to reject it.

As far as the lunar tide is concerned, consider first that the lunar orbit lies in a plane that is inclined only by very few degrees with respect to the ecliptic plane. Hence, as a first order approximation, we can safely assume that the Moon can be considered as lying in the ecliptic plane. The Moon, however, in addition to an approximately periodical recurrence of

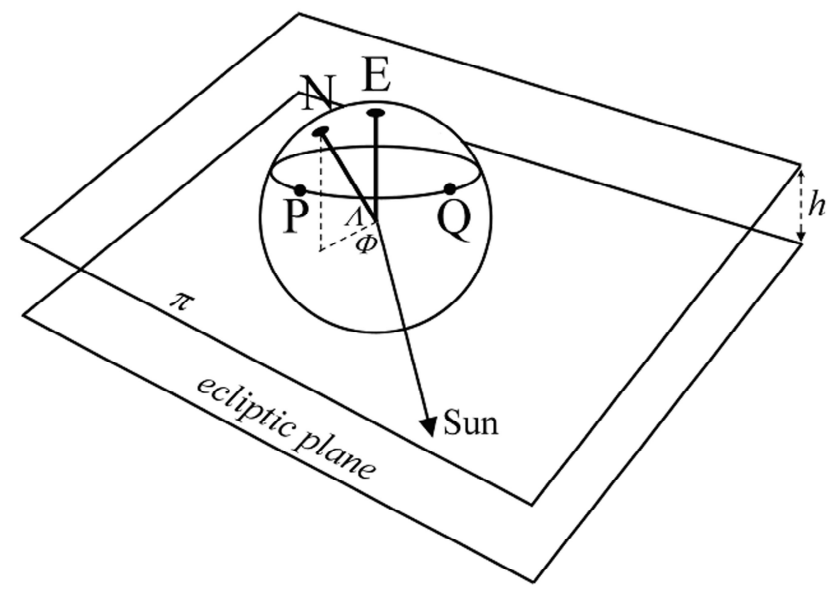

Fig. 10. An approximate computation of the seasonal variation of the solar loading tide on the Pacific coast of Eurasia. Consider two planes, i.e. the ecliptic plane, and the plane $\pi$ parallel to it and located at a distance $h$. The sphere is the Earth's surface. The North Pole is denoted by $N$, and the perpendicular to the ecliptic plane crosses the Earth's surface at $E$. The point $P$ is along the Eurasian coast, and the point $Q$ is along the North American coast. It is supposed that the loading tide that pushes on the continental shelf at $P$ is proportional to the linear extension of the arc $P Q$ along the circle that is the intersection of $\pi$ with the Earth's surface. The total loading tide on Eurasia is an integral of such effect for every point along the Eurasian coast. The lunar effect is computed in a much similar way, where however the direction of the Sun has to be substituted by the position of the Moon. The solar variation gives a seasonal oscillation, the lunar variation gives a $\sim 29$ days period oscillation. At some large distance, the shorter period oscillation is damped off due the greater plastic behaviour compared to the seasonal variation. Hence, the final result is that one should observe a seasonal variation of the stress propagating through the crust.

29 days, has a very complicated orbit and, in particular, one should consider the Meton cycle of $\sim 18.6$ years, by which the aforementioned modelling applied to the solar tide ought to be repeated upon considering the actual position of the Moon on every specific date of every given year. Differently stated, let us suppose that we do have available records from the Italian peninsula and from Cephallonia lasting for, say, 20 years or longer. By such database we should detect the Meton period in our $H F A E$ records, much like by our much shorter database we could recognise an annual modulation.

The lunar loading tide, however, can be modelled even by a few years database, by means of some suitable astronomical computation, etc. In any case, it appears reasonable guessing that the apparent large scatter observed in our figures, which shows the annual modulation, is the likely result of the solar loading tide. In fact, the general background trend is of solar origin, while the superposed regular, although large, modulation is caused by the lunar loading tide, which should display a period of, say, 29 days, that was neglected while considering the annual modulation alone. The 
annual modulation is the result of the regularity of the Earth's orbit around the Sun. In contrast, an equivalent regularity dealing with the lunar loading tide ought to display the $~ 29$ days periodicity and the Meton cycle.

The concern shifts therefore on the discussion of the large physical scatter of the observed $H F A E$ records compared to the "smooth" annual variation in the background. If such scatter results from the lunar loading tide, such scatter must behave according to the Meton cycle.

However, such interpretation can have a different observational check. In fact, consider the stray electric currents that are induced into the Earth's body (ocean water, sediments, crust, lithosphere, etc.) by the electromagnetic signals impinging from space. For the following items refer to the detailed review and discussion given by Meloni et al. (1983) and by Lanzerotti and Gregori (1986), where detailed references are given. A classical and well-known effect is the skin depth phenomenon. In fact, shorter wavelengths penetrate to comparatively much shallower depths, as the induced electric currents produce a Faraday screening that forbids penetration into the deeper layers. That is, the electromagnetic induction is definitely a concern affecting only, and almost exclusively, some comparatively much shallow layers of the Earth's body. In principle, their effects ought to influence the spin rate of the Earth. Nevertheless, such influence operates on some shallow layers, thus implying some stress propagation through the Earth's crust and through its entire body.

In fact, the state-of-the-art concerning the observed variations of the 1.o.d. can be synthesised, in some simple minded though expressive way, upon distinguishing basically three classes of phenomena. (i) One phenomenon is the main trend, which is believed being associated with the lunar (and with the lesser solar) tide, i.e., with the aforementioned loading tide (some subtle concern, however, was raised by some specialists, although such aspect is not relevant for the present discussion). Some apparently noisy disturbances appear superposed over such regular and very smooth trend. Their physical significance was debated for some long time, until some evidence clearly showed that they are not random noise. Let us distinguish, very roughly, between, say, some first order and second order perturbations. (ii) The second aforementioned phenomenon is such second order perturbations, that were shown by Hide et al. (1980) being correlated with the time changes of the total angular momentum of the atmosphere (in fact, a comparatively larger angular momentum of the atmosphere must imply, by total angular momentum conservation, a slowing down of the spin rate of the Earth, and viceversa). A similar correlation was later observed being associated with the similar action played by the total angular momentum of ocean water. However, some comparatively larger effects on the l.o.d. remain unexplained, which were tout court briefly called here above "first order" perturbations (iii), which are the third aforementioned phenomenon. A reasonable guess is that the transient, and apparently erratic, perturbations, caused by the time varying electromagnetic induced current within the Earth, play a role similar to what occurs inside the electromagnetic brakes of a train. That is, the stray currents affect the spin rate of the Earth.

In principle, such effect can be measured by means of the standard geomagnetic records available for the international networks of observatories. However, as it happens when dealing with such very tiny and sporadic effects, some severe practical bias derives for the error propagation resulting (i) from the limited and uneven distribution of the geomagnetic observatories on the globe, (ii) from the lack of observatories in oceanic areas, and (iii) from the fact that the few available oceanic observatories are located on tiny islands, which are very often volcanic, and which are per se a violent anomaly concerning the underground structure of electrical conductivity. They are therefore severely biased when they are used for investigating the induced currents within the Earth. That is, the geomagnetic database appears very poorly suited for such kind of investigation. The situation can be substantially ameliorated upon considering the induced potential voltages within the planetary network of the submarine communication cables. In fact, their substantial practical advantage relies on the fact that, unlike a geomagnetic observatory that gives only a point-like and local measurement, they provide us with a signal integrated over some long spatial range (in the order of several thousand kilometre linear extension). This permits putting some constraints and thus controlling the devastating role played by error propagation. Such investigation, which was proposed by Meloni et al. (1983) was, however, not yet carried out.

In the case that such analysis becomes eventually available, and that it results successful in the fact that it gets rid of error propagation etc., the scatter of the annual variation of the $H F A E$ observations ought to result, on the one hand very regular for the part that is to be associated with the Meton cycle of the lunar loading tide, and, on the other hand, it should reflect the apparently erratic modulation deriving from the electromagnetic stray currents that affect the spin rate of the Earth. In fact, such stray currents affect mainly the comparatively much shallower layers of the Earth's body, and therefore, while affecting the spin rate of the entire Earth, they must originate some crustal stress.

The entire leading concern is evidently about the sensitivity of every specific recording device being considered, about the precision of its records - and such precision is referred not only to the sensors, to the electronics, to the data acquisition system, etc., rather to the entire monitoring system represented by natural Earth's structures - and about the robustness of the needed algorithms and computations for carrying out the data handling. All such items cannot be discussed a priori with any actual reference to some actual observational database. The entire problem appears intriguing, having potential applications for the understanding of the stress regime within the crust, and for its implications for the precursors of earthquakes, etc. 


\section{Conclusions}

The $H F A E$ reflects behaviour of the crustal materials, when they are comparatively less affected by the fatigue of their solid components, and they appear more akin to an elastic object. Under such circumstances, a regular response of the crust is observed, correlated within the Italian peninsula and at Cephallonia, with no relative time-shift. This fact envisages a likely astronomical modulation, appearing therefore a planetary phenomenon, which in fact is manifested like an annual variation of a stress regularly crossing the Mediterranean area.

When the role of fatigue enters into play - and this occurs up to some minor extent for the $H F A E$, and much more effectively for the $L F A E$ records - evidence is found of crustal stress propagation crossing the region of the $A E$ recording site. The evidence appears just like in the case of solitons crossing through the area being monitored. Such phenomenon ought to be identified as an individual event, crossing through different areas, thus envisaging a concrete possibility of measuring the propagation speed of such stress soliton through the Earth, on continental or planetary scale. Such measure requires, however, availability of $H F A E$ and $L F A E$ records in some array of recording stations.

Such much regular features, however, appear variously perturbed by, and intermingled with, the influence originated by the specific tectonic setting of every given region being considered. In such a respect, the Italian peninsula and the area of the Cephallonia Island have much different morphologies, and display correspondingly different behaviours with respect to crustal stress, $A E$ monitoring, and seismicity.

For getting final confirmation of the interpretation here proposed of the observed phenomena, an array of $A E$ recording stations ought to be recommended, being located on a regional to continental scale.

The possibility is discussed that the clear annual variation observed, being in phase when comparing the Italian peninsula and Cephallonia, could be the consequence of the annual variation experienced by the loading tide, associated with the different inclination of the rotation axis of the Earth, by which the water of the Pacific ocean ought to respond to the tidal pull according to a seasonal modulation.

Such speculative and tentative interpretation can be tested either by means of astronomical modelling, beginning from the average trend implied by the solar tide, or by some much more detailed modelling that should take into account the astronomical motion of the Moon, with its 29 days periodicity, and, on a much longer time scale, with the Meton cycle. In addition, some lesser transient effects could be eventually evidenced, upon considering the expected influence on the length of the day (1.o.d.), originated by the erratic stray electric currents within the Earth, caused by the time varying electromagnetic signals impinging from space. Such last effect, however, could be seriously hampered by the error propagation while performing data handling of the geomagnetic records from the international network of observatories, and the induced voltages within submarine communication cables, perhaps, could result particularly useful for a practical management of such entire analysis.

Acknowledgements. The authors express their gratitude to A. Raponi and A. M. Raponi for kindly hosting the $A E$ monitoring apparatus in the basement of their house at Orchi (Italy) and to the Greek colleagues who have been collecting the $A E$ data in Cephallonia. Moreover, the authors are grateful to M. Lupieri for his contribution in fixing the plots in Figs. 1 to 7.

Edited by: P. F. Biagi

Reviewed by: K. M. Storetvedt

\section{References}

Gregori, G. P.: Galaxy-Sun-Earth relations. The origin of the magnetic field and of the endogenous energy of the Earth, with implications for volcanism, geodynamics and climate control, and related items of concern for stars, planets, satellites, and other planetary objects. A discussion in a prologue and two parts, Beiträge zur Geschichte der Geophysik und Kosmischen Physik, Band 3, Heft 3, 471 pp., 2002.

Gregori, G. P. and Paparo, G.: Acoustic emission (AE). A diagnostic tool for environmental sciences and for non destructive tests (with a potential application to gravitational antennas), in: Meteorological and geophysical fluid dynamics, edited by: Schröder, W., Arbeitkreis Geschichte der Geophysik und Kosmische Physik, Science Edition, Bremen, 166-204, 2004.

Gregori, G. P., Paparo, G., Poscolieri, M., and Zanini, A.: Acoustic Emission and Released Seismic Energy, Nat. Hazards Earth Syst. Sci., 5, 777-782, 2005, http://www.nat-hazards-earth-syst-sci.net/5/777/2005/.

Hide, R., Birch, N. T., Morrison, L. V., Shea, D. J., and White, A. A.: Atmospheric angular momentum fluctuations and changes in the length of the day, Nature, 286, 114-117, 1980.

Lanzerotti, L. J. and Gregori, G. P.: Telluric currents: the natural environment and interactions with man-made systems, in: The Earth's electrical environment, edited by: Krider, E. P. and Roble, R. G., National Academy Press, Washington D.C., 232257, 1986.

Meloni, A., Lanzerotti, L. J., and Gregori, G. P.: Induction of currents in long submarine cables by natural phenomena, Rev. Geophys. Space Phys., 21(4), 795-803, 1983.

Paparo, G. and Gregori, G. P.: Multifrequency acoustic emissions $(A E)$ for monitoring the time evolution of microprocesses within solids, Reviews of quantitative nondestructive evaluation, 22, in: AIP Conference Proceedings, edited by: Thompson, D. O. and Chimenti, D. E., 1423-1430, 2003.

Paparo, G., Gregori, G. P., Poscolieri, M., Marson, I., Angelucci, F., and Glorioso, G.: Crustal stress crises and seismic activity in the Italian peninsula investigated by fractal analysis of acoustic emission, soil exhalation and seismic data, in: Fractal Analysis for Natural Hazards, edited by: Cello, G. and Malamud, B. D., Geological Society, London, Special Publications, 261, 47-61, 2006. 
Paparo, G., Gregori, G. P., Coppa, U., De Ritis, R., and Taloni, A.: Acoustic Emission $(A E)$ as a diagnostic tool in geophysics, Annls Geophys., 45(2), 401-416, 2002.

Poscolieri, M., Lagios, E., Gregori, G. P., Paparo, G., Sakkas, V. A., Parcharidis, I., Marson, I., Soukis, K., Vassilakis, E., Angelucci, F., and Vassilopoulou, S.: Crustal stress and seismic activity in the Ionian archipelago as inferred by combined satelliteand ground-based observations, Kefallinìa, Greece, in: Fractal Analysis for Natural Hazards, edited by: Cello, G. and Malamud, B. D., Geological Society, London, Special Publications, 261, 63-78, 2006
Wunsch, C.: Moon, tides and climate, Nature, London, 405, 743744,2000 